\title{
Subject Index Vol. 66, 1996
}

Accuracy 137 Alginate 141

Arteriovenous malformation 41 Autoclave time 141 Automatic surface alignment 69

Cell transplantation 141

Cerebral arteriovenous malformations 1

Chemical shift 118

Chronic pain 19,65

Computed tomography 58

Computer-assisted spine surgery 108

Cortical mapping 24

Craniotomy guidance 81

Distortion 123,137

Extracranial stereotactic radiosurgery 1

Frameless localization 75 -stereotaxy 91 -surgery 69 Functional disorders 19 -MRI 24 -neurosurgery 65

Gamma Knife ${ }^{\circledR} 19,35,41$ — radiosurgery 15 
- unit facility 41

Image-directed neurosurgery 96 Image-guided registration 69 Intensity modulation 30 Interactive image guidance 108 Intracranial tumor 41 Intraoperative imaging 58

Laser localization 75 LINAC 1,10

Magnetic neurosurgery 102

- resonance imaging 58,118,123,137

Malfunction 35

Meningioma 15 Microencapsulation 141 Microscope, stereotactic 96 Minimally invasive procedure 102 Motor cortex 65 MR distortion 84

Neuronavigation 65

Nucleus ventralis intermedius 65

Parkinson's disease 19 Peacock treatment 30 Pedicle screw fixation 108

(C) 1996 S. Karger AG, Basel 1011-6125/96/0663-0015 \$10.00/0 
Physically based model 84

Radiosurgery 1,19,24,30,35,41,58 Radiotherapy 10

Rectification 137 Remote manipulation 102 Robotic arm 75

Spatial shift 118 Spinal metastases 1 Spine surgery 108 Stereovideo-based tracking 81 
Stereotactic neurosurgery 84,123 -surgery 58 -techniques 10,91 Stereotaxis 1

Target guidance 91 Trajectory guidance 91 Treatment 15 Tremor 65 Trigeminal neuralgia 19

Warping 84 